\title{
Quantification of Nitric Oxide and Reactive Oxygen Species in Leishmania-infected J774A.1 Macrophages as a Response to the in vitro treatment with a Natural Product Compound Kalliopi Karampetsou, ${ }^{1,2, \#}$, Olga S. Koutsoni ${ }^{1, \#}$ and Eleni Dotsika ${ }^{1, *}$
}

${ }^{1}$ Laboratory of Cellular Immunology \& National Reference Laboratory for Leishmaniasis, Department of Microbiology, Hellenic Pasteur Institute, 127 Vass. Sofias av., 11521 Athens, Greece; ${ }^{2 D i v i s i o n ~ o f ~}$ Pharmacognosy and Natural Products Chemistry, Department of Pharmacy, National and Kapodistrian University of Athens, Panepistimiopolis Zografou, 15771 Athens, Greece

*For correspondence: e.dotsika@pasteur.gr

\#Contributed equally to this work

[Abstract] Leishmaniasis is a parasitic disease caused by the obligatory intracellular protozoa Leishmania spp. Current therapeutic options are limited and thus, drug discovery against leishmaniasis is very important. Nevertheless, there is a great difficulty to develop therapeutic strategies against the disease because the parasite deploys various mechanisms to evade the immune system and multiply inside the host. Among the main factors of the immunity that are recruited to confront the Leishmania infection are the macrophages (MФs) that produce effector molecules such as Nitric Oxide (NO) and Reactive Oxygen Species (ROS). Therefore, efficient drug agents should combine the antileishmanial effect of these gaseous transmitters along with the enhancement of the host's adaptive immunity. In the quest of therapeutic alternatives, natural products have been extensively studied and are considered as candidate antileishmanial agents since they exhibit specific properties in modulating the host's immune response towards an effective anti-leishmanial cell-mediated immunity capable to eliminate parasitic dissemination. In the current protocol, Leishmania-infected MФs (J774A.1 cell line) that have been treated with various increasing concentrations of a natural compound, are tested for the production of the aforementioned molecules. In order to detect NO production, we employ the Griess colorimetric nitrite assay and quantification relies on the construction of an accurate standard curve using appropriate standards of known concentration. ROS detection and quantification is achieved by flow cytometry and relies on the use of carboxy- $\mathrm{H}_{2}$ DCFDA, an indicator that converts to a fluorescent form when interacts with ROS molecules.

Keywords: Nitric Oxide, Reactive Oxygen Species, Innate immunity, Immune mechanisms, Natural products, Leishmania spp., Macrophages

[Background] Macrophages (MФs) are important innate immune effector cells that participate in host defense, providing enhanced antimicrobial activity. They are characterized by a remarkable plasticity and unique abilities to polarize toward different phenotypes. On one hand, classically activated macrophages are known to have major roles in host defense against various microbial pathogens, while alternatively activated macrophages are instrumental in immune regulation and wound healing (Leopold Wager and Wormley, 2014). The term "classically" activated is used to designate the effector 
macrophages that are produced during cell-mediated immune responses upon the dual signal of interferon- $\gamma$ (IFN- $\gamma$ ) and tumor-necrosis factor (TNF), characterized by enhanced microbicidal or tumoricidal capacity and secretion of high levels of pro-inflammatory cytokines and immune mediators. More specifically, IFN-Y is produced by innate (NK cells) or adaptive immune cells (T helper 1 cells) and primes macrophages to secrete pro-inflammatory cytokines and to produce increased amounts of superoxide anions, oxygen and nitrogen radicals that increase their killing ability (Dale et al., 2008).

The obligatory intracellular parasites of the genus Leishmania have deployed sophisticated mechanisms to evade and modulate the host's immune system for their benefit and consequently, they are able to survive and persist within MФs. Consequently, in the case of Leishmania infection, MФs are having a dual role either as effector cells with leishmanicidal activity or as host cells. The fate of Leishmania spp. parasites is determined by the activation status of MФs (Tomiotto-Pellissier et al., 2018). "Classically" activated MФs are capable of killing parasites effectively via the production of Nitric Oxide (NO) upon activation of inducible nitric oxide synthase (iNOS) and other leishmanicidal molecules, such as Reactive Oxygen Species (ROS). NO and ROS are two key players in the macrophage defense system against intracellular parasites (Horta et al., 2012; Roy et al., 2017). Nevertheless, Leishmania spp. parasites have been adapted to survive and replicate in this hostile environment by deploying antioxidant systems or by suppressing ROS and NO production (Kumar et al., 2001; Denkers and Butcher, 2005; Gupta et al., 2013).

In recent years, there is an increasing interest in the study of natural products against leishmaniasis because several plant extracts or isolated compounds exhibit a promising antileishmanial activity which may not only be due to their direct action on parasite but also to a concomitant effect on the host's immune response (Rodrigues et al., 2015). Several natural products have been tested for their ability to increase the production of ROS and/or NO in in vitro and in vivo experimental models of leishmaniasis (Torres-Santos et al., 1999; do Socorro et al., 2003; Georgopoulou et al., 2007; Patricio et al., 2008).

In this protocol, we aim to quantify the NO and ROS production by Leishmania spp.-infected J774A.1 macrophages as a response to treatment with various increasing concentrations of Total Phenolic Fraction (TPF) derived from Extra Virgin Olive Oil (Angelis et al., 2017). The quantification of NO is achieved with the use of Griess assay which relies on the accumulation of nitrites in cell culture supernatants. Respectively, the relative quantification of TPF-mediated ROS generation is determined with the use of cell-permeable carboxy- $\mathrm{H}_{2}$ DCFDA fluorescent probe which is deacetylated by cellular esterases to form 2',7'-dichlorodihydrofluorescein ( $\left.\mathrm{H}_{2} \mathrm{DCF}\right)$. In the presence of ROS, predominantly $\mathrm{H}_{2} \mathrm{O}_{2}, \mathrm{H}_{2} \mathrm{DCF}$ is rapidly oxidized to $2^{\prime}, 7^{\prime}$-dichlorofluorescein (DCF), which is highly fluorescent, with excitation and emission wavelengths of 498 and $522 \mathrm{~nm}$, respectively (Bae et al., 2000). These protocols can be used to gain a first in vitro evidence, about whether or not, these immune mechanisms are activated by an antimicrobial agent.

\section{Materials and Reagents}

1. Cell Culture Flasks with filter cap, $25 \mathrm{~cm}^{2}$ (Thermo Fisher Scientific, catalog number: 156367) 
2. Cell culture Flasks, plug seal cap, $25 \mathrm{~cm}^{2}$ (Greiner Bio-One, catalog number: 690160)

3. Cell scrapers (Sarstedt, catalog number: 83.1830)

4. Cover glasses square (VWR, catalog number: 6311570)

5. Falcon ${ }^{\circledR}$ Round-Bottom Tubes, $5 \mathrm{ml}$ (Corning, catalog number: 352008$)$

6. 96-well flat bottom tissue culture plates (Sarstedt, catalog number: 83.3924.005)

7. 24-well flat bottom tissue culture plates (Sarstedt, catalog number: 83.3922.005)

8. Pipettes tips: $0.5-10 \mu \mathrm{l}, 10-200 \mu \mathrm{l}, 200-1,000 \mu \mathrm{l}$ (Greiner Bio-One, catalog numbers: 771291, 739290, 740290)

9. Pleated filter paper (Sigma-Aldrich, catalog number: WHA1201150)

10. Reaction tubes, $1.5 \mathrm{ml}$ (Greiner Bio-One, catalog number: 616201)

11. Serological pipettes $2 \mathrm{ml}, 5 \mathrm{ml}, 10 \mathrm{ml}$ (Sarstedt, catalog numbers: 86.1252.001, 86.1253.001, 86.1254.001)

12. Sterile syringe filter $0.22 \mu \mathrm{m}$ (Millipore, catalog number: SLGVV255F)

13. Syringes $5 \mathrm{ml}$ (Terumo, catalog number: SS+05S21381)

14. Cell lifter (Corning, catalog number: 3008 )

15. Leishmania infantum promastigotes (zymodeme GH8, strain MHOM/GR/2001/GH8)

16. Leishmania major promastigotes (zymodeme LV39, strain MRHO/SU/59/P)

17. Immortalized macrophage cell line J774A.1 (ATCC; Rockville, USA/ ATCC No: TIB-67)

18. Total Phenolic Fraction (derived from Extra Virgin Olive Oil from agricultural cooperative in Zaros region, Crete, Greece)

19. RPMI-1640 (w/o) L-glutamine (Biowest, catalog number: L0501)

20. Fetal Bovine Serum (Biowest, catalog number: S181B)

21. L-glutamine (Biosera, catalog number: LM-R1641)

22. HEPES buffer $1 \mathrm{M}$ (Biowest, catalog number: L0180)

23. Penicillin-Streptomycin solution $10,000 \mathrm{U} / \mathrm{ml}$ (Biowest, catalog number: L0022)

24. Dimethyl sulfoxide (DMSO) Cell culture grade (PanReac Applichem, catalog number: A3672,0050)

25. Ethanol absolute (Sigma-Aldrich, catalog number: 32205-M)

26. Formalin (Sigma-Aldrich, catalog number: R04586-82)

27. Trypan blue dye for vital staining (BDH, catalog number: 34078 )

28. Milteforan ${ }^{\circledR} 20 \mathrm{mg} / \mathrm{ml}$ (Hexadecylphosphocholine, [HePC], Virbac S.A.)

29. Hydrochloric acid $(\mathrm{HCl})$ solution, $1 \mathrm{M}$ (Sigma-Aldrich, catalog number: 150696)

30. Sodium chloride $99.9 \%(\mathrm{NaCl})$ (Applichem, catalog number: 381659 )

31. Potassium chloride (KCl), ACS reagent, $\geq 99.0 \%$ (Sigma-Aldrich, catalog number: 746336)

32. Sodium phosphate dibasic $\left(\mathrm{Na}_{2} \mathrm{HPO}_{4}\right)$, ACS reagent, $\geq 99.0 \%$ (Sigma-Aldrich, catalog number: 795410)

33. Potassium phosphate monobasic $\left(\mathrm{KH}_{2} \mathrm{PO}_{4}\right)$, ACS reagent, $\geq 99.0 \%$ (Sigma-Aldrich, catalog number: 795488)

34. Sodium hydroxide ( $\mathrm{NaOH}$ ) solution, $1 \mathrm{M}$ (Sigma-Aldrich, catalog number: 79724) 
35. Carboxy- $\mathrm{H}_{2}$ DCFDA (Thermo Fisher Scientific, catalog number: C400)

36. Hydrogen Peroxide solution $30 \%$ (w/w) (Sigma-Aldrich, catalog number: H1009)

37. ortho-Phosphoric acid $85 \%$ (AppliChem, catalog number: A0989)

38. Sulfanilamide (Sigma-Aldrich, catalog number: S9251-100G)

39. N-(1-Naphthyl) ethylenediamine dihydrochloride (Sigma-Aldrich, catalog number: 222488)

40. Sodium nitrite $\left(\mathrm{NaNO}_{2}\right)$ (Sigma-Aldrich, catalog number: 237213)

41. Complete RPMI-1640 medium (see Recipes)

42. Fetal Bovine Serum solution (FBS) (see Recipes)

43. Phosphate Buffer Saline (PBS), 10x, pH 7.2-7.4 (see Recipes)

44. $0.4 \%(w / v)$ Trypan blue exclusion dye (see Recipes)

45. Sodium nitrite $\left(\mathrm{NaNO}_{2}\right)$ solution (see Recipes)

46. Griess reagent (see Recipes)

47. $\mathrm{H}_{2} \mathrm{O}_{2}$ solution (see Recipes)

\section{Equipment}

1. New Brunswick ${ }^{\mathrm{TM}}$ Galaxy ${ }^{\circledR} 170 \mathrm{~S} \mathrm{CO}_{2}$ Incubator (Eppendorf, catalog number: Galaxy $170 \mathrm{~S}$ )

2. Refrigerated Incubator $26^{\circ} \mathrm{C}$ (Sanyo, catalog number: MIR-253)

3. BD FACSCalibur Flow Cytometer: 3-Color (BD FACSCalibur ${ }^{\mathrm{TM}}$, catalog number: 342973 )

4. ELISA Microplate reader (Dynatech Laboratories, catalog number: MRX)

5. Optical microscope (Olympus, catalog number: BHB)

6. Sterile biosafety cabinet (Telstar, catalog number: Bio-II-A)

7. Gilson pipettes (Gilson, catalog numbers: PIPETMAN Classic P-10, P-20, P-200, P-1000)

8. Malassez counting chamber (Paul Marienfeld $\mathrm{GmbH} \&$ Co., catalog number: 0640610)

9. Multichannel pipette (Brand, catalog number: 703710)

10. $\mathrm{pH}$ Meter (Thermo Fisher Scientific, catalog number: 13-644-928)

11. Pipette controller (Brand, catalog number: accu-jet ${ }^{\circledR}$ pro 26300)

12. Water distiller (Sartorius, catalog number: H2O-I-1-UV-T)

\section{Software}

1. Microsoft ${ }^{\circledR}$ Office Excel 2010 (Microsoft)

2. FlowJo, version $10(\mathrm{BD})$

\section{Procedure}

A. In vitro quantification of nitric oxide production by Leishmania-infected J774A.1 macrophage treated with increasing concentrations of TPF 
1. Prepare a known concentration of the plant extract using the appropriate solvent. TPF is dissolved in $62.5 \%$ pure ethanol, $31.25 \%$ sterile distilled water and $6.25 \%$ DMSO.

Note: The recovery of TPF from extra virgin olive oil was carried out by Centrifugal Partition Extraction (CPE) technique, which is an innovative solid support free separation technique derived from Centrifugal Partition Chromatography (CPC). Briefly, liquid-liquid chromatography was performed using a laboratory scale centrifugal partition extractor $F C P E 300^{\circledR}$, which was equipped with a rotor composed of 7 stacked partition disks engraved with a total of 231 partition cells, while the total volume of the column was $300 \mathrm{ml}$ (Angelis et al., 2017; Koutsoni et al., 2018).

2. Cultivate J774A.1 macrophage in $25 \mathrm{~cm}^{2}$ cell culture flasks with filter cap containing $10 \mathrm{ml}$ of complete RPMI-1640 medium (Recipe 1) at $37{ }^{\circ} \mathrm{C}$ under $5 \% \mathrm{CO}_{2}$ humidified air.

3. Allow macrophages to reach a high density population of about $70 \%$ confluence.

Note: J774A.1 macrophages cultured in complete RPMI-1640 medium at $37^{\circ} \mathrm{C}$ under $5 \% \mathrm{CO}_{2}$ humidified air, usually reach $70 \%$ confluence within 3 to 4 days.

4. Then, remove the majority of culture medium with a sterile disposable transfer pipette and leave about $2 \mathrm{ml}$ medium in the flask and detach macrophage monolayer by scrapping cells gently and slowly with a cell scraper at $45^{\circ}$ angle.

5. Determine the number of macrophages per $\mathrm{ml}$ by differential counting of dead and live cells using the Trypan blue exclusion dye (Recipe 4) in a Malassez counting chamber under an optical microscope.

Note: The final dilution of macrophages in Trypan blue dye depends on the density of the culture. Usually, a 1:20 final dilution of macrophages is suitable at the $3^{\text {rd }}$ or $4^{\text {th }}$ day of the in vitro culture.

6. Seed $5 \times 10^{4} \mathrm{~J} 774 \mathrm{~A} .1$ macrophages per well in a final volume of $100 \mu \mathrm{l}$ of complete RPMI-1640 medium in a 96-well flat bottom tissue culture plate under a sterile biosafety cabinet.

7. Incubate the plate for $18 \mathrm{~h}$ at $37^{\circ} \mathrm{C}$ under $5 \% \mathrm{CO}_{2}$ humidified air in order to achieve cell adhesion.

8. Determine the number of Leishmania spp. promastigotes per $\mathrm{ml}$ (promastigotes must be in their stationary phase of growth) by differential counting of dead and live parasites using the Trypan blue exclusion dye in a Malassez counting chamber under an optical microscope.

Notes:

a. Leishmania spp. promastigotes usually enter the stationary-growth phase after 3 to 5 days, depending on the Leishmania strain. For example, L. major, a commonly used strain, approximately enters the stationary growth phase at Day 4 when reaches the number of 3.5 $x 10^{7}$ parasites $/ \mathrm{ml}$ (Nasiri et al., 2013).

b. The final dilution of promastigotes in Trypan blue dye depends on the density of the in vitro parasite culture. Usually, a 1:20 final dilution of promastigotes is suitable at the $3^{\text {rd }}$ or $4^{\text {th }}$ day of the in vitro culture. Additionally, promastigotes have to be fixed with $2 \% \mathrm{v} / \mathrm{v}$ formalin before counting.

9. Seed $7.5 \times 10^{5}$ Leishmania spp. early stationary phase promastigotes in each well (i.e., ratio of 15:1 parasites/macrophage) in a total final volume of $200 \mu$ l of complete RPMI-1640 medium. 
10. Incubate the plate for $48 \mathrm{~h}$ at $37^{\circ} \mathrm{C}$ under $5 \% \mathrm{CO}_{2}$ humidified air.

11. Then, remove the non-internalized promastigotes by washing thrice with RPMI-1640 medium pre-warmed at $37^{\circ} \mathrm{C}$.

12. Add various increasing concentrations of TPF $(5-400 \mu \mathrm{g} / \mathrm{ml})$ in triplicates and fill in the wells with complete RPMI-1640 medium until the final volume of $200 \mu$ per well.

Note: Concentrations of plant extracts may vary depending on the natural product.

13. Add one reference drug (i.e., one drug currently subscribed for the chemotherapy of leishmaniasis) in triplicate at the appropriate $50 \%$ inhibitory concentration ( $\left(\mathrm{C}_{50}\right)$. Hexadecylphosphocholine (HePC) is used at the concentrations of $0.6 \mu \mathrm{g} / \mathrm{ml}$ and $3.2 \mu \mathrm{g} / \mathrm{ml}$ for L. infantum and L. major respectively, as previously described (Koutsoni et al., 2018).

Note: Control triplicates of the equivalent volumes of TPF solvents must also be included.

14. Triplicate of a negative control group must also be included. More specifically, a triplicate of Leishmania-infected J774A.1 macrophages cultured only in the presence of complete RPMI1640 medium without any drug influence, serves as a negative control group.

15. Incubate the plate for $48 \mathrm{~h}$ at $37^{\circ} \mathrm{C}$ under $5 \% \mathrm{CO}_{2}$ humidified air.

16. Collect culture supernatants and transfer $50 \mu \mathrm{l}$ of each sample into a new 96 -well flat bottom tissue culture plate, as designated in Figure 1. It is also recommended to use again triplicates for each sample.

Note: Culture supernatants contain the secreted reactive nitrogen intermediates.

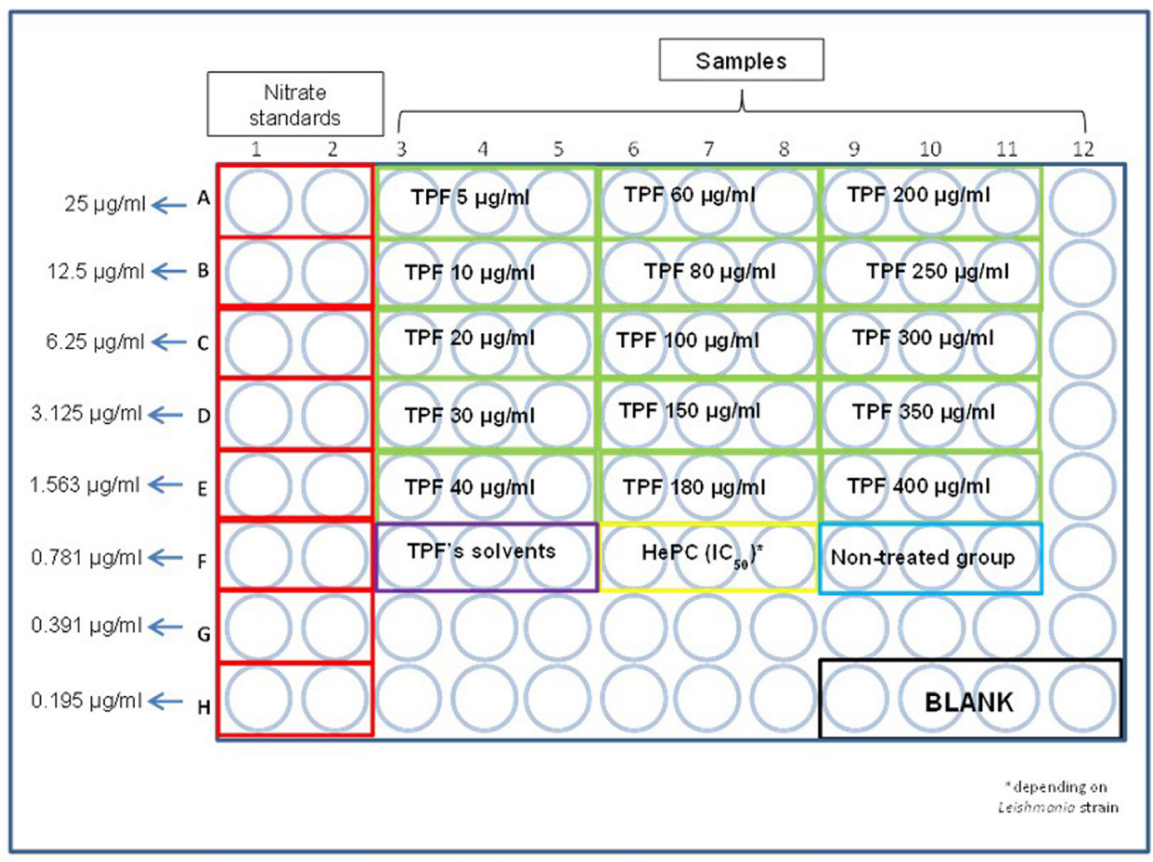

Figure 1. Representative illustration of the 96-well flat bottom plate that Griess assay takes place 
17. A sodium nitrite standard curve is required. Place $100 \mu \mathrm{l}$ of $\mathrm{NaNO}_{2}$ solution (Recipe 5) at concentration of $75 \mu \mathrm{g} / \mathrm{ml}$ at $\mathrm{A} 1$ and $\mathrm{A} 2$ wells, as designated in Figure 1.

18. Place $50 \mu$ of distilled $\mathrm{H}_{2} \mathrm{O}$ in $\mathrm{B} 1, \mathrm{~B} 2$ etc. until $\mathrm{H} 1$ and $\mathrm{H} 2$ wells and perform serial dilutions by transferring $50 \mu \mathrm{l}$ of $\mathrm{NaNO}_{2}$ solution from $\mathrm{A} 1$ and $\mathrm{A} 2$ to $\mathrm{B} 1$ and $\mathrm{B} 2$ respectively until $\mathrm{H} 1$ and $\mathrm{H} 2$, with the use of a multichannel pipette.

Note: Discard the excessive $50 \mu$ obtained from $\mathrm{H} 1$ and $\mathrm{H} 2$ wells.

19. Place $100 \mu \mathrm{l}$ of Griess reagent (Recipe 6) in both standards and samples subsequently. Notes:

a. Griess reagent must be freshly prepared. When preparing Griess reagent, avoid contact with the bottleneck because this usually contains Griess-positive material that gives an increase in background absorbance.

b. The final standard $\mathrm{NaNO}_{2}$ concentrations range from $0.195 \mu \mathrm{g} / \mathrm{ml}$ to $25 \mu \mathrm{g} / \mathrm{ml}$.

c. A quadruplicate that contains only $50 \mu$ of distilled water that serves as blank is also included.

20. Read the plate by using an absorbance microplate reader with excitation at $570 \mathrm{~nm}$.

Note: Color change from transparent to pink is immediately noticed. The intensity of the color is greater as nitrite concentration gets higher.

B. In vitro quantification of reactive oxygen species production by Leishmania-infected J774A.1 macrophages treated with increasing concentrations of TPF

1. Prepare a known concentration of the plant extract using the appropriate solvent. TPF is dissolved in $62.5 \%$ pure ethanol, $31.25 \%$ sterile distilled water and $6.25 \%$ DMSO.

Note: The recovery of TPF from extra virgin olive oil was carried out by Centrifugal Partition Extraction (CPE) technique, which is an innovative solid support free separation technique derived from Centrifugal Partition Chromatography (CPC), as mentioned in Procedure section, Step A1.

2. Cultivate J774A.1 macrophages in $25 \mathrm{~cm}^{2}$ cell culture flasks with filter cap, containing $10 \mathrm{ml}$ of complete RPMI-1640 medium, as previously described in Step A2.

3. Allow macrophages to reach a high density population of about $70 \%$ confluence, as mentioned in Step A3.

4. Then, determine the number of macrophages per ml by differential counting of dead and live cells using the Trypan blue exclusion dye in a Malassez counting chamber under an optical microscope, as already described in Steps A4 and A5.

5. Seed $5 \times 10^{5} \mathrm{~J} 774 \mathrm{~A} .1$ macrophages per well in a final volume of $500 \mu \mathrm{l}$ of complete RPMI-1640 medium in a 24-well flat bottom tissue culture plate under a sterile biosafety cabinet (Figure 2). 


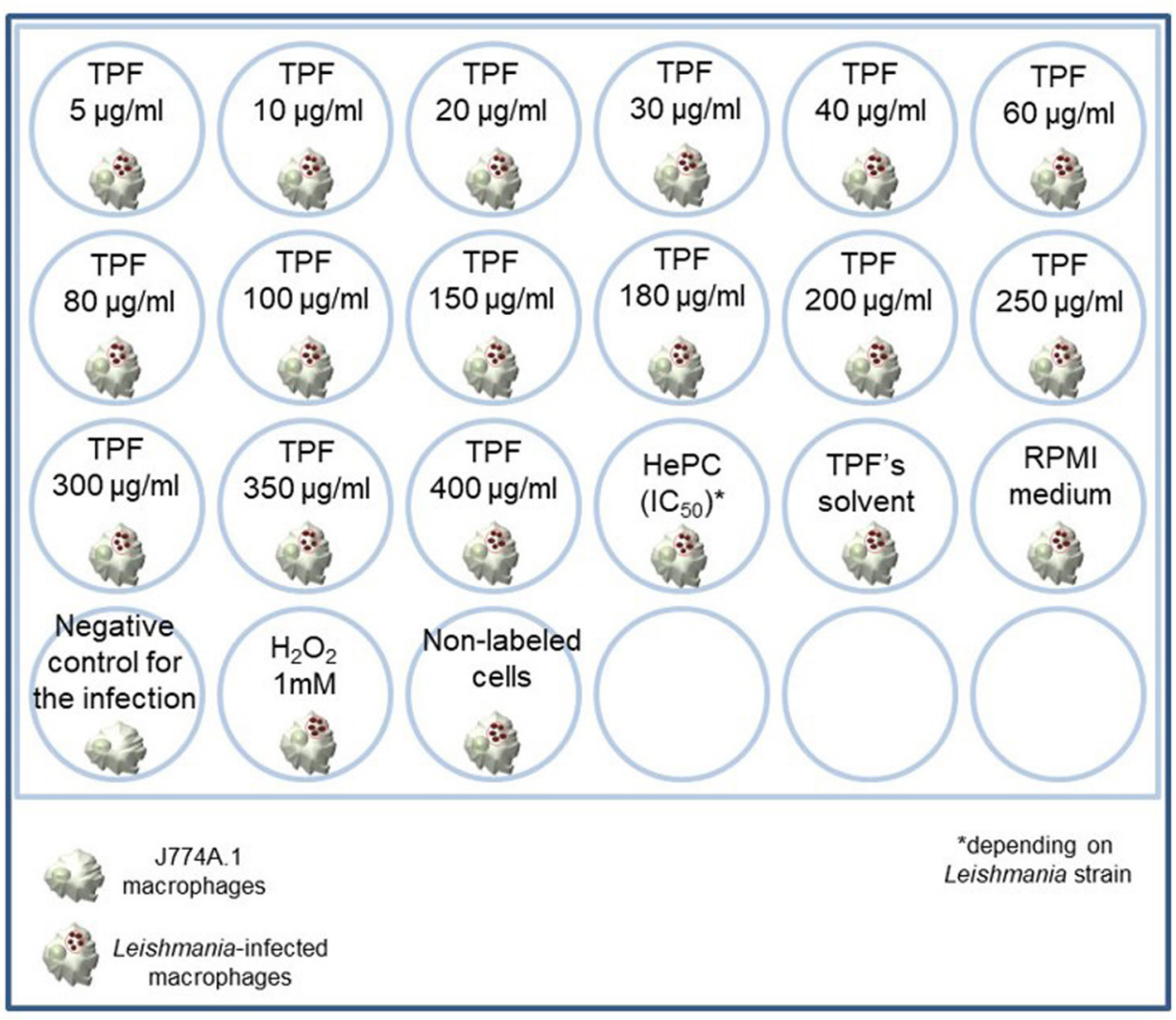

Figure 2. Representation of the 24-well tissue culture plate

6. Incubate the plate for $18 \mathrm{~h}$ at $37^{\circ} \mathrm{C}$ under $5 \% \mathrm{CO}_{2}$ humidified air in order to achieve cell adhesion.

7. Determine the number of Leishmania spp. promastigotes per $\mathrm{ml}$ (promastigotes must be in their stationary phase of growth) by differential counting of dead and live parasites using the Trypan blue exclusion dye in a Malassez counting chamber under an optical microscope.

Notes:

a. Leishmania spp. promastigotes usually enter the stationary-growth phase after 3 to 5 days, depending on the Leishmania strain. For example, L. major, approximately enters the stationary growth phase at Day 4 when reaches the number of $3.5 \times 10^{7}$ parasites $/ \mathrm{ml}$ (Nasiri et al., 2013).

b. The final dilution of promastigotes in Trypan blue dye depends on the density of the in vitro parasite culture. Usually, a 1:20 final dilution of promastigotes is suitable at the $3^{\text {rd }}$ or $4^{\text {th }}$ day of the in vitro culture. Additionally, promastigotes have to be fixed with $2 \% \mathrm{v} / \mathrm{v}$ formalin before counting.

8. Seed $7.5 \times 10^{6}$ Leishmania spp. early stationary phase promastigotes in each well (i.e., ratio of 15:1 parasites/macrophage) in a total final volume of $1,000 \mu$ l of complete RPMI-1640 medium. Note: Infection negative control group is included and is consisted of promastigote-free macrophages.

9. Incubate the plate for $48 \mathrm{~h}$ at $37^{\circ} \mathrm{C}$ under $5 \% \mathrm{CO}_{2}$ humidified air. 
10. Then, remove the non-internalized promastigotes by washing thrice with RPMI-1640 medium pre-heated at $37^{\circ} \mathrm{C}$.

11. Add various increasing concentrations of TPF $(5-400 \mu \mathrm{g} / \mathrm{ml})$ and fill in the wells with complete RPMI-1640 medium until the final volume of $1,000 \mu \mathrm{l}$ per well.

Note: Concentrations of plant extracts may vary depending on the natural product.

12. Add one reference drug (i.e., one drug currently subscribed for the chemotherapy of leishmaniasis) in triplicate at the appropriate $50 \%$ inhibitory concentration $\left(\mathrm{IC}_{50}\right)$. Hexadecylphosphocholine (HePC) is used at the concentrations of $0.6 \mu \mathrm{g} / \mathrm{ml}$ and $3.2 \mu \mathrm{g} / \mathrm{ml}$ for L. infantum and L. major respectively, as previously described (Koutsoni et al., 2018).

Notes:

a. Control of the equivalent volumes of TPF solvents must also be included.

b. Three different control groups must be included i) Leishmania spp. infected-macrophages that will serve as treatment negative control, ii) Leishmania spp. infected-macrophages that will serve later as positive control for ROS production and iii) Leishmania spp. infectedmacrophages that will serve later in the flow cytometry as the non-labeled sample.

13. Incubate the plate for $48 \mathrm{~h}$ at $37^{\circ} \mathrm{C}$ under $5 \% \mathrm{CO}_{2}$ humidified air.

14. Remove culture supernatants and add $500 \mu \mathrm{l}$ of PBS $1 \mathrm{x}$ solution (Recipe 3 ) in each well except from the positive control for ROS production where you add $500 \mu \mathrm{l}$ of $1 \mathrm{mM} \mathrm{H}_{2} \mathrm{O}_{2}$ solution (Recipe 7).

Note: $\mathrm{H}_{2} \mathrm{O}_{2}$ is an inducer of ROS production by the macrophages and therefore is useful as positive control for the ROS production (Ogawa et al., 2004).

15. Incubate the plate for $15 \mathrm{~min}$ at $37^{\circ} \mathrm{C}$ under $5 \% \mathrm{CO}_{2}$ humidified air.

16. Remove the culture supernatant from the positive control group and replace with $500 \mu$ l of PBS $1 \mathrm{x}$ solution.

17. Add $5 \mu \mathrm{M}$ of the carboxy- $\mathrm{H}_{2}$ DCFDA in each well.

Note: In the non-labeled group, avoid adding the carboxy- $\mathrm{H}_{2} \mathrm{DCFDA}$.

18. Incubate the plate for $30 \mathrm{~min}$ at $37^{\circ} \mathrm{C}$ under $5 \% \mathrm{CO}_{2}$ humidified air.

19. Remove the culture supernatants and add in each well $500 \mu \mathrm{l}$ of PBS $1 \mathrm{x}$ solution.

20. Detach cells with the use of a cell lifter.

21. Collect one by one cell suspensions and transfer them into a $5 \mathrm{ml}$ round bottom tube each.

Note: Tubes containing cell suspensions should be placed on ice during the procedure of the flow cytometric data collection.

22. Analyze the samples in the FACSCalibur.

\section{Data analysis}

A. In vitro quantification of NO production by Leishmania-infected J774A.1 macrophages treated with increasing concentrations of TPF 
Griess colorimetric nitrite assay relies on the conversion of the nitric oxide in a sample to a stable azo compound that absorbs at around $540 \mathrm{~nm}$ (Sun et al., 2003). Put the plate in the microplate reader in order to obtain the Optical Density (OD) value of each well.

1. Calculate the mean Optical Density (OD) value of the blank quadruplicate and subtract it from every other OD value.

2. Calculate the mean of the $O D$ values for each of the standard nitrite concentrations.

3. Select all the values that correspond to the standard nitrite concentrations and insert a scatter plot that depicts the nitrite concentration in the x-axis and the mean of the corresponding $O D$ values in the $y$-axis.

4. Extract the linear region of the curve.

5. Select "add trendline" and pick "linear" trendline options.

6. Add the trendline in the chart and extract the equation and R-squared value of the trendline (Figure 3.).

Note: A trendline is most reliable when its $R$-squared value is at or near 1 .

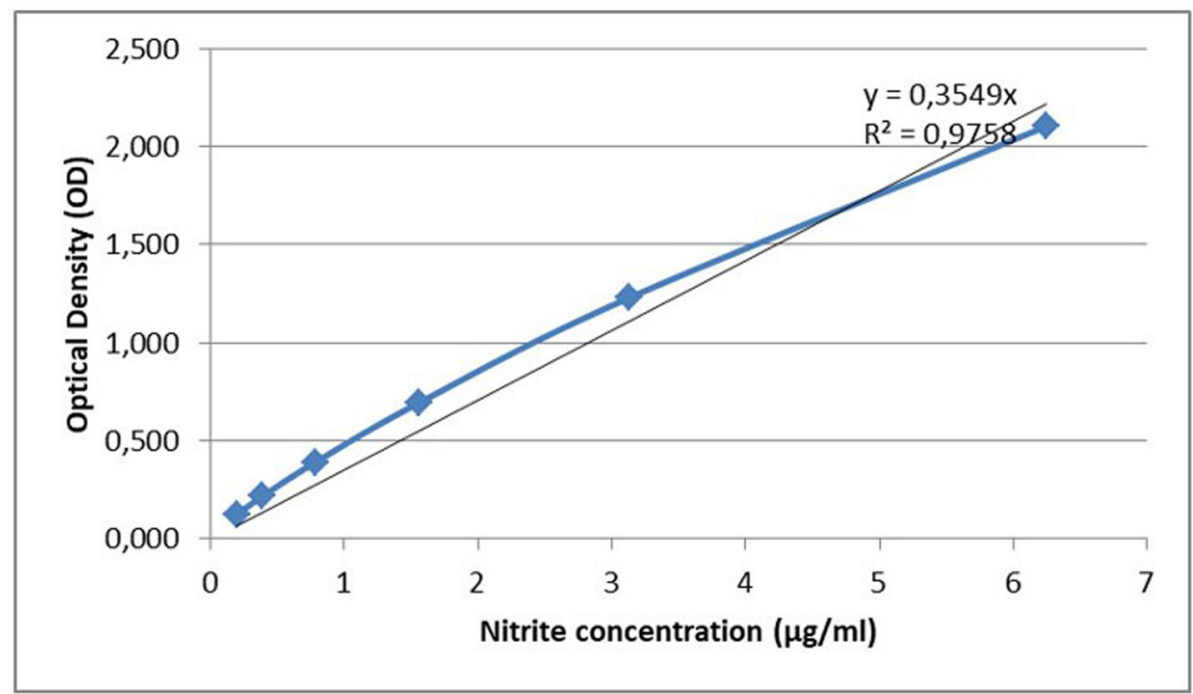

Figure 3. Linear region of the standard curve with trendline

7. Calculate the mean of the OD values that corresponds to each sample.

8. Based on the equation, find the nitrite concentration for each sample.

B. In vitro quantification of ROS production by Leishmania-infected J774A.1 macrophages treated with increasing concentrations of TPF

For the data analysis, FlowJo software is used.

1. Analyze the samples according to their size (forward-scattered light/ FSC) and cell granularity (side-scattered light/ SSC) on a dual parameter dot plot and set a gating region to exclude cellular debris (Figure 4).

Note: As a general guide, cellular debris is FSC-low. 


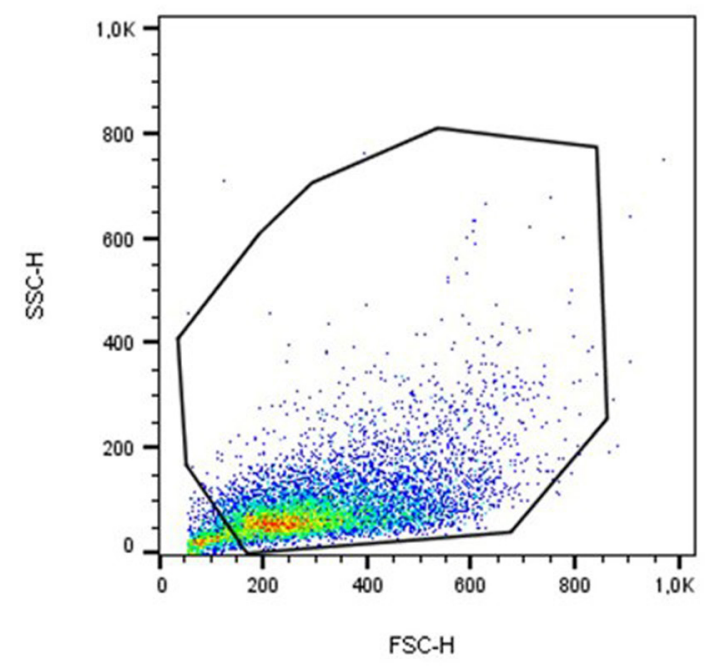

Figure 4. An example of gating J774A.1 macrophages analyzed by FSC and SSC dot plot

2. Analyze the gated cell population in a single-parameter histogram (FL1-H channel).

3. Extract the Geo-mean for each sample (Figure 5).

Note: Geo-mean is representative of the fluorescence intensity of the sample. The greater the Geo-mean, the more is the fluorescence intensity and consequently the more is the presence of ROS in the cells (Eruslanov and Kusmartsev, 2010).
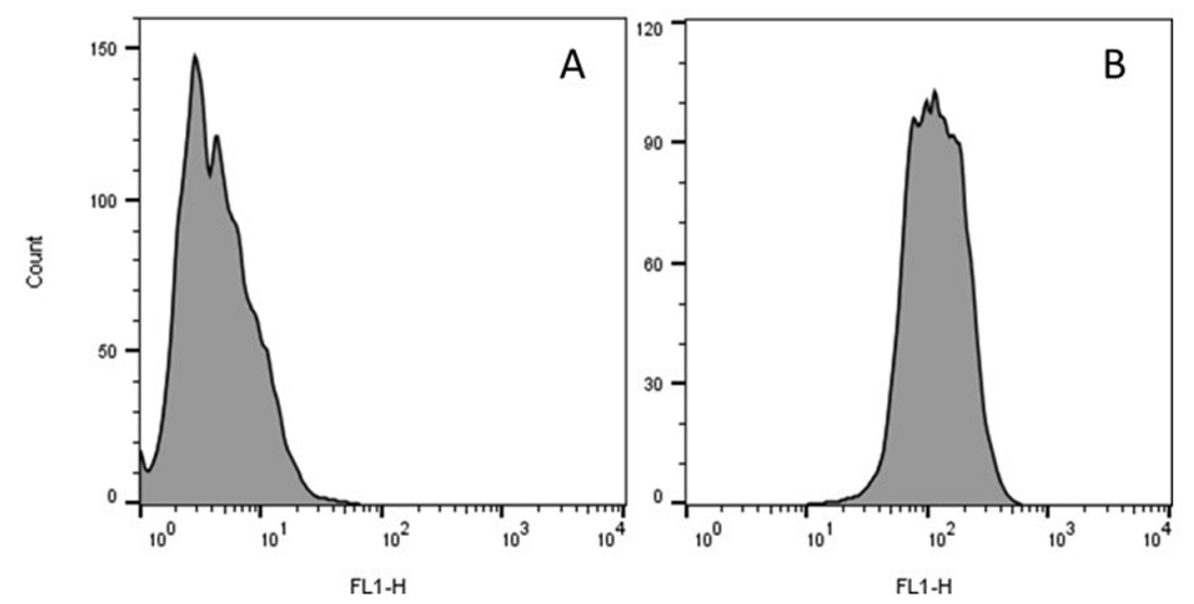

Figure 5. Representative single-parameter histograms of flow-cytometric analysis for the quantification of ROS production. FL1-histogram of the fluorescence intensity (Geo Mean) in gated population of J774A. 1 cells loaded with $\mathrm{H}_{2}$ DCFDA upon stimulation with (A) RPMI-1640 medium and (B) TPF at $400 \mu \mathrm{g} / \mathrm{ml}$.

\section{$\underline{\text { Recipes }}$}

1. Complete RPMI-1640 medium 
a. Add $5 \mathrm{ml}$ of L-glutamine (stock solution $200 \mathrm{mM}$, stored at $-20{ }^{\circ} \mathrm{C}$ ), $5 \mathrm{ml}$ of PenicillinStreptomycin (stock solution $10,000 \mathrm{U} / \mathrm{ml}$, stored at $-20{ }^{\circ} \mathrm{C}$ ) and $5 \mathrm{ml}$ of HEPES (stock solution $1 \mathrm{M}$ ) to $500 \mathrm{ml}$ RPMI-1640 medium

b. Store at $4{ }^{\circ} \mathrm{C}$

c. Take the appropriate volume and add FBS to $10 \%(\mathrm{v} / \mathrm{v})$ final concentration

2. Fetal Bovine Serum solution (FBS)

Thaw FBS and heat-inactivate it at $56{ }^{\circ} \mathrm{C}$ for $30 \mathrm{~min}$ in a water-bath under constant agitation Store at $-20^{\circ} \mathrm{C}$ until use

3. Phosphate Buffer Saline (PBS), 10x, pH 7.2-7.4

a. Place $800 \mathrm{ml}$ of distilled water in a suitable container and dissolve $80 \mathrm{~g} \mathrm{NaCl}, 2 \mathrm{~g} \mathrm{KCl}, 11.5$ $\mathrm{g}$ of $\mathrm{Na}_{2} \mathrm{HPO}_{4}$, and $2 \mathrm{~g}$ of $\mathrm{KH}_{2} \mathrm{PO}_{4}$. Agitate until the complete salt dilution and adjust solution to the desired $\mathrm{pH}$ (typically 7.2-7.4) with $\mathrm{NaOH}(1 \mathrm{M})$ or $\mathrm{HCl}(1 \mathrm{M})$ solution, if needed

b. Add distilled water until the final volume is $1,000 \mathrm{ml}$ and sterilize through a $0.22 \mu \mathrm{m}$ pore size syringe filter unit

c. Prepare the $1 \mathrm{x}$ working solution by diluting $50 \mathrm{ml}$ of $10 \mathrm{x}$ stock solution in $450 \mathrm{ml}$ of sterile and distilled water. Store at $4{ }^{\circ} \mathrm{C}$

4. $0.4 \%(w / v)$ Trypan blue exclusion dye

a. Dissolve $0.4 \mathrm{~g}$ of Trypan blue in $100 \mathrm{ml}$ of PBS 1x. Agitate well

b. Filter through a pleated filter paper and sterilize through a $0.22 \mu \mathrm{m}$ pore size syringe filter unit. Store at room temperature

5. Sodium nitrite $\left(\mathrm{NaNO}_{2}\right)$ solution

Prepare a stock solution of $75 \mu \mathrm{g} / \mathrm{ml}$

Dissolve $750 \mu \mathrm{g}$ in $10 \mathrm{ml}$ of de-ionized water

6. Griess reagent

Griess reagent consists of two separate solutions ( $A$ and $B$ ) that they are mixed together (ratio 1:1) at the moment of the experiment operation

\section{Griess reagent A}

First, prepare a $3 \%(\mathrm{v} / \mathrm{v})$ solution of phosphoric acid. Then, dissolve the appropriate amount of sulfanilamide at a final concentration of $1 \%(\mathrm{w} / \mathrm{v})$

\section{Griess reagent B}

Dissolve the appropriate amount of $\mathrm{N}$-(1-Naphthyl) ethylenediamine dihydrochloride in distilled water in order to obtain a final concentration of $0.1 \%(\mathrm{w} / \mathrm{v})$

7. $\mathrm{H}_{2} \mathrm{O}_{2}$ solution
a. Prepare $100 \mathrm{ml}$ working solution of $1 \mathrm{mM}$
b. Dissolve $10 \mu \mathrm{l}$ of stock solution $(30 \% \mathrm{w} / \mathrm{w})$ in $100 \mathrm{ml}$ of de-ionized water 


\section{Acknowledgments}

Part of this research work was supported by the Hellenic Foundation for Research and Innovation (HFRI) and the General Secretariat for Research and Technology (GSRT), under the HFRI Ph.D. Fellowship grant (GA. no. $6 \mathrm{~N} \Delta \Theta 46 \Psi \mathrm{Z} 2 \mathrm{~N}-\Sigma \Sigma \mathrm{O}$ ). Also, this work was supported by KRHPIS II (MIS 5002486) and EATRIS (MIS 5028091).

The protocols were adapted and modified by Koutsoni et al., 2018 and Kyriazis et al., 2016.

\section{Competing interests}

Authors declare that they have no competing interests.

\section{$\underline{\text { References }}$}

1. Angelis, A., Hamzaoui, M., Aligiannis, N., Nikou, T., Michailidis, D., Gerolimatos, P., Termentzi, A., Hubert, J., Halabalaki, M., Renault, J. H. and Skaltsounis, A. L. (2017). An integrated process for the recovery of high added-value compounds from olive oil using solid support free liquidliquid extraction and chromatography techniques. J Chromatogr A 1491: 126-136.

2. Bae, Y. S., Sung, J. Y., Kim, O. S., Kim, Y. J., Hur, K. C., Kazlauskas, A. and Rhee, S. G. (2000). Platelet-derived growth factor-induced $\mathrm{H}_{2} \mathrm{O}_{2}$ production requires the activation of phosphatidylinositol 3-kinase. J Biol Chem 275(14): 10527-10531.

3. Dale, D. C., Boxer, L. and Liles, W. C. (2008). The phagocytes: neutrophils and monocytes. Blood 112(4): 935-945.

4. Denkers, E. Y. and Butcher, B. A. (2005). Sabotage and exploitation in macrophages parasitized by intracellular protozoans. Trends Parasitol 21(1): 35-41.

5. do Socorro, S. R. M. S., Mendonca-Filho, R. R., Bizzo, H. R., de Almeida Rodrigues, I., Soares, R. M., Souto-Padron, T., Alviano, C. S. and Lopes, A. H. (2003). Antileishmanial activity of a linalool-rich essential oil from Croton cajucara. Antimicrob Agents Chemother 47(6): 1895-1901.

6. Eruslanov, E. and Kusmartsev, S. (2010). Identification of ROS using oxidized DCFDA and flowcytometry. Methods Mol Biol 594: 57-72.

7. Georgopoulou, K., Smirlis, D., Bisti, S., Xingi, E., Skaltsounis, L. and Soteriadou, K. (2007). In vitro activity of 10-deacetylbaccatin III against Leishmania donovani promastigotes and intracellular amastigotes. Planta Med 73(10): 1081-1088.

8. Gupta, G., Oghumu, S. and Satoskar, A. R. (2013). Mechanisms of immune evasion in leishmaniasis. Adv Appl Microbiol 82: 155-184.

9. Horta, M. F., Mendes, B. P., Roma, E. H., Noronha, F. S., Macedo, J. P., Oliveira, L. S., Duarte, M. M. and Vieira, L. Q. (2012). Reactive oxygen species and nitric oxide in cutaneous leishmaniasis. J Parasitol Res 2012: 203818. 
10. Koutsoni, O. S., Karampetsou, K., Kyriazis, I. D., Stathopoulos, P., Aligiannis, N., Halabalaki, M., Skaltsounis, L. A. and Dotsika, E. (2018). Evaluation of total phenolic fraction derived from extra virgin olive oil for its antileishmanial activity. Phytomedicine 47: 143-150.

11. Kumar, R., Pai, K. and Sundar, S. (2001). Reactive oxygen intermediates, nitrite and IFNgamma in Indian visceral leishmaniasis. Clin Exp Immunol 124(2): 262-265.

12. Kyriazis, I. D., Koutsoni, O. S., Aligiannis, N., Karampetsou, K., Skaltsounis, A. L. and Dotsika, E. (2016). The leishmanicidal activity of oleuropein is selectively regulated through inflammation- and oxidative stress-related genes. Parasit Vectors 9: 441.

13. Leopold Wager, C. M. and Wormley, F. L., Jr. (2014). Classical versus alternative macrophage activation: the Ying and the Yang in host defense against pulmonary fungal infections. Mucosal Immunol 7(5): 1023-1035.

14. Nasiri, V., Karimi, G., Dalimi, A., Paykari, H. and Ghaffarifar, F. (2013). Effects of sheep and mouse urine on the growth pattern of Leishmania major promastigotes. Biomed Res Int 2013: 748592.

15. Ogawa, Y., Kobayashi, T., Nishioka, A., Kariya, S., Ohnishi, T., Hamasato, S., Seguchi, H. and Yoshida, S. (2004). Reactive oxygen species-producing site in hydrogen peroxide-induced apoptosis of human peripheral T cells: involvement of lysosomal membrane destabilization. Int J Mol Med 13(3): 383-388.

16. Patricio, F. J., Costa, G. C., Pereira, P. V., Aragao-Filho, W. C., Sousa, S. M., Frazao, J. B., Pereira, W. S., Maciel, M. C., Silva, L. A., Amaral, F. M., Rebelo, J. M., Guerra, R. N., Ribeiro, M. N. and Nascimento, F. R. (2008). Efficacy of the intralesional treatment with Chenopodium ambrosioides in the murine infection by Leishmania amazonensis. $J$ Ethnopharmacol 115(2): 313-319.

17. Rodrigues, I. A., Mazotto, A. M., Cardoso, V., Alves, R. L., Amaral, A. C., Silva, J. R., Pinheiro, A. S. and Vermelho, A. B. (2015). Natural products: insights into leishmaniasis inflammatory response. Mediators Inflamm 2015: 835910.

18. Roy, S., Dutta, D., Satyavarapu, E. M., Yadav, P. K., Mandal, C., Kar, S. and Mandal, C. (2017). Mahanine exerts in vitro and in vivo antileishmanial activity by modulation of redox homeostasis. Sci Rep 7(1): 4141.

19. Sun, J., Zhang, X. J., Broderick, M. and Fein H. (2003). Measurement of nitric oxide production in biological systems by using Griess Reaction assay. Sensors 3(8): 276-284.

20. Tomiotto-Pellissier, F., Bortoleti, B., Assolini, J. P., Goncalves, M. D., Carloto, A. C. M., MirandaSapla, M. M., Conchon-Costa, I., Bordignon, J. and Pavanelli, W. R. (2018). Macrophage polarization in leishmaniasis: broadening horizons. Front Immunol 9: 2529.

21. Torres-Santos, E. C., Moreira, D. L., Kaplan, M. A., Meirelles, M. N. and Rossi-Bergmann, B. (1999). Selective effect of 2',6'-dihydroxy-4'-methoxychalcone isolated from Piper aduncum on Leishmania amazonensis. Antimicrob Agents Chemother 43(5): 1234-1241. 